Research Article

\title{
The Reliability of the Use of Serum Neutrophil Gelatinase-Associated Lipocalin Levels in the Assessment of Renal Functions after Coronary Artery Bypass Grafting
}

\author{
Ozan Onur Balkanay (D), Deniz Göksedef, Suat Nail Ömeroğlu, and Gökhan İpek \\ Cerrahpasa Medical Faculty, Department of Cardiovascular Surgery, Istanbul University, Istanbul, Turkey \\ Correspondence should be addressed to Ozan Onur Balkanay; balkanay@doctor.com
}

Received 19 October 2017; Revised 9 January 2018; Accepted 4 February 2018; Published 5 March 2018

Academic Editor: Luigina Guasti

Copyright (C) 2018 Ozan Onur Balkanay et al. This is an open access article distributed under the Creative Commons Attribution License, which permits unrestricted use, distribution, and reproduction in any medium, provided the original work is properly cited.

\begin{abstract}
Objective. Evaluation of perioperative renal function is very important for early diagnosis and treatment of acute kidney injury after coronary artery bypass grafting. Serum creatinine levels, creatinine clearance, and estimated glomerular filtration rates used in determination of postoperative kidney injury can lead to late detection. Therefore, it is necessary to make a diagnosis earlier in clinical practice and to search for a reliable method. The reliability of the use of serum neutrophil gelatinase-associated lipocalin levels in close follow-up of renal function was evaluated in patients with coronary artery bypass grafting under cardiopulmonary bypass in our study. Patients and Methods. A total of 40 patients who underwent coronary artery bypass grafting under cardiopulmonary bypass between September 2009 and February 2010 were included in the study. The reliability of the postoperative 1st day plasma neutrophil gelatinase-associated lipocalin (Triage ${ }^{\circledast}$ NGAL Device; Biosite Inc.) measurements was evaluated in kidney injury developed in the first 5 days after operation that was detected using the Risk-Injury-Failure-Loss-End stage criteria. Results. Ten (25\%) women and $30(75 \%)$ male patients were included in the study. The average age is $59 \pm 8.6$ years. Kidney injury according to Risk-Injury-Failure-Loss-End stage criteria developed in 8 patients (20\%). For $150 \mathrm{ng} / \mathrm{mL}$ cutoff value of postoperative plasma neutrophil gelatinase-associated lipocalin levels, the area under the receiver-operating characteristic curve was 0.965 . Neutrophil gelatinase-associated lipocalin's sensitivity, specificity, and negative and positive predictive values were $100 \%$, $93.8 \%, 100 \%$, and $80 \%$, respectively. Conclusion. It has been determined that plasma neutrophil gelatinase-associated lipocalin levels can be reliably used for early diagnosis of kidney dysfunction in patients undergoing coronary artery bypass grafting.
\end{abstract}

\section{Introduction}

One of the leading complications following cardiac surgery is acute kidney injury [1]. Cardiopulmonary bypass (CPB) is the main factor in this complication. In multicenter studies, cardiac surgery performed under $\mathrm{CPB}$ is shown as the second most common cause of acute kidney injury (AKI) after nephrotoxic drug use $[2,3]$. AKI after cardiac surgery is known to increase postoperative mortality and morbidity [4-7]. The main reason for this poor prognosis is that early detection is not possible and effective early treatment is not performed $[8,9]$. Serum creatinine level measurements for long years have been used as the main indicator of kidney dysfunction [5]. However, the use of serum creatinine levels in the early detection of acute kidney injury is less sensitive [5]. Serum creatinine levels can be normal even if there is over $50 \%$ damage in the kidneys [5]. There is a study showing that $41.1 \%$ of the cases can be underdiagnosed by means of AKI according to the serum creatinine level [8]. Additionally, the reliability of the estimated glomerular filtration rate (GFR) measurements to indicate the creatinine clearance rate in patients undergoing coronary artery bypass grafting (CABG) is suboptimal [10]. For these reasons, new researches have been conducted and new biomarkers, which can detect early kidney damage and enable early intervention, continued to be investigated. Recent studies indicate that measurement of the neutrophil gelatinaseassociated lipocalin (NGAL) level is one of the early 
biomarkers that can be used to detect CPB-associated AKI $[5,11]$. The aim of our study was to investigate the reliability of the use of serum NGAL levels in the early detection of AKI after CABG under CPB.

\section{Patients and Methods}

A total of 40 consecutive patients without preexisting renal failure undergoing CABG under CPB between September 2009 and February 2010 were included in the study, who did not have exclusion criteria and were operated by the same surgical team. The kidney injury during the 5-day follow-up period was determined according to the Risk-Injury-FailureLoss-End stage (RIFLE) criteria, creatinine clearance rate measurements, and estimated glomerular filtration rate measurements according to the Cockcroft-Gault equation, and the reliability of the postoperative first day plasma NGAL values for renal dysfunction was retrospectively investigated [12].

In the RIFLE criteria, there are 5 subcategories of kidney dysfunction: risk (1.5-fold increased serum creatinine levels or GFR decrease higher than $25 \%$ or urine output lower than $0.5 \mathrm{~mL} / \mathrm{kg} / \mathrm{h}$ during 6 hours), injury (2-fold increased serum creatinine levels or GFR decrease higher than $50 \%$ or urine output lower than $0.5 \mathrm{~mL} / \mathrm{kg} / \mathrm{h}$ during 12 hours), failure (3-fold increased creatinine levels higher than or equal to $4 \mathrm{mg} / \mathrm{dL}$ or acute rise higher than or equal to $0.5 \mathrm{mg} / \mathrm{dL}$ or GFR decrease of $75 \%$ or urine output lower than $0.3 \mathrm{~mL} / \mathrm{kg} / \mathrm{h}$ during 24 hours or anuria during 12 hours), loss (persistent acute renal failure and complete loss of renal function more than 4 weeks), and ESRD (end-stage renal disease more than 3 months) [12].

Concomitant surgical procedures, off-pump surgery, presence of the history of antithrombotic agent usage during 2 weeks preoperatively, presence of accompanying acute infection, presence of chronic infection, presence of preoperative advanced liver or pulmonary insufficiency, and preexisting renal failure were considered among the exclusion criteria.

2.1. Acute Kidney Injury. The definition of AKI is used as a general definition for acute kidney dysfunction [5]. Acute Dialysis Quality Initiative identifies AKI within the RIFLE criteria $[12,13]$. In our study, kidney injury was detected according to the RIFLE criteria [12]. The RIFLE classification includes both glomerular filtration rate (GFR) and urine output (UO) criteria. Injury category is defined as a 2-fold increase in serum creatinine level or GFR decrease higher than $50 \%$ or UO lower than $5 \mathrm{~mL} / \mathrm{kg} / \mathrm{h}$ during 12 hours.

2.2. Plasma Neutrophil Gelatinase-Associated Lipocalin Measurement. The NGAL measurements were performed at the time of intensive care follow-up at 24 hours after termination of CPB using the Triage NGAL Test Device (Biosite Inc., San Diego, CA, USA). The cutoff value was accepted as $150 \mathrm{ng} / \mathrm{mL}$ in the measurements.
2.3. Statistical Analysis. Categorical variables were expressed as frequency and percentage, and continuous variables were expressed as mean \pm standard deviation (95\% confidence interval). $P$ value of less than 0.05 was considered significant. IBM SPSS software package version 21.0 (SPSS, Chicago, IL, USA) was used for statistical analysis. The receiver-operating characteristic (ROC) curve was drawn to calculate the area under the curve (AUC). Values above 0.7 for AUC-ROC were considered to have good test performance. Sensitivity, specificity, and positive and negative predictive values of serum NGAL measurements for detecting AKI were calculated.

2.4. Primary End Point. The calculation of the confidence of the serum NGAL measurement based on the $150 \mathrm{ng} / \mathrm{mL}$ cutoff value of the blood sample taken at 24 hours following termination of the CPB in detecting AKI according to the RIFLE criteria was accepted as the primary end point of the study.

\section{Results}

Ten $(25 \%)$ women and 30 (75\%) male patients were included in the study. The average age was $58.7 \pm 8.6(56-61.2)$ years (Table 1). The mean body mass index of the patients was $28.5 \pm 4.3(27.3-30) \mathrm{kg} / \mathrm{m}^{2}$, and the mean body surface area was $1.9 \pm 0.2(1.8-1.9) \mathrm{m}^{2}$ (Table 1). A total of 35 patients (87.5\%) had hypertension, 26 patients $(65 \%)$ had hyperlipidemia, and 16 patients (40\%) had diabetes mellitus. All patients had CABG indication and underwent elective onpump CABG surgery. None of them had a preoperative kidney dysfunction history.

The mean \pm standard deviation $(95 \% \mathrm{CI})$ values of creatinine clearance rates on preoperative and postoperative 1st and 5 th days were $85.7 \pm 30$ (76.9-94.5), $109.4 \pm 42$ (97.8$121.8)$, and $87.8 \pm 32.8(77.7-97.7) \mathrm{mL} \cdot \mathrm{min}^{-1} \cdot 1.73 \mathrm{~m}^{2}$, respectively (Table 2). Kidney injury according to the RIFLE criteria was developed in 8 patients (20\%) during the postoperative 5-day period. At the $150 \mathrm{ng} / \mathrm{mL}$ cutoff value of the postoperative first day plasma NGAL level, the AUCROC was 0.965 (Figure 1). The sensitivity, specificity, and negative and positive predictive values of NGAL on the postoperative first day in predicting the kidney injury in the period of postoperative 5 days were found as $100 \%, 93.8 \%$, $100 \%$, and $80 \%$, respectively.

\section{Discussion}

There is an intense relationship between on-pump CABG and the development of the postoperative kidney injury. It is stated that AKI can be developed at high levels up to $30-50 \%$ after open-heart surgery under CPB $[5,14,15]$. However, kidney failure requiring dialysis is observed in approximately $1-2 \%$ of patients after cardiac surgery, and this is associated with about $60 \%$ mortality $[16,17]$. The leading risk factors contributed to develop AKI after open-heart surgery under $\mathrm{CPB}$ are preoperative kidney dysfunction, advanced age, hypertension, diabetes mellitus, impaired left ventricular ejection fraction, perioperative hemodynamic instability, 
TABle 1: Perioperative patient variables.

\begin{tabular}{|c|c|c|c|c|c|}
\hline Variable & $n$ & $\%$ & Mean \pm SD & Min.-max. & $95 \% \mathrm{CI}$ \\
\hline \multicolumn{6}{|l|}{ Preoperative } \\
\hline Age (year) & & & $58.7 \pm 8.6$ & $40-79$ & $56-61.2$ \\
\hline Female gender $(\mathrm{F})$ & 10 & 25 & & & \\
\hline Body mass index $\left(\mathrm{kg} / \mathrm{m}^{2}\right)$ & & & $28.5 \pm 4.3$ & $23-42$ & $27.3-30$ \\
\hline Body surface area $\left(\mathrm{m}^{2}\right)$ & & & $1.9 \pm 0.2$ & $1.5-2.3$ & $1.8-1.9$ \\
\hline Hypertension & 35 & 87.5 & & & \\
\hline Hyperlipidemia & 26 & 65 & & & \\
\hline Diabetes mellitus & 16 & 40 & & & \\
\hline \multicolumn{6}{|l|}{ Intraoperative } \\
\hline Aortic cross clamping time ( $\mathrm{min})$ & & & $60.2 \pm 28.1$ & $12-110$ & $51.2-68.7$ \\
\hline Total perfusion time (min) & & & $92.4 \pm 34.9$ & $35-151$ & $81.8-102.9$ \\
\hline Distal bypass & & & $2 \pm 0.9$ & $1-4$ & $1.8-2.3$ \\
\hline \multicolumn{6}{|l|}{ Postoperative } \\
\hline Extubation time (hour) & & & $14 \pm 14.4$ & 4-95 & $10.7-18.9$ \\
\hline ICU follow-up (hour) & & & $49.5 \pm 19.1$ & $40-120$ & $44.2-55.9$ \\
\hline Kidney injury* & 8 & 20 & & & \\
\hline Hospital stay (day) & & & $8.4 \pm 3$ & $6-19$ & $7.6-9.4$ \\
\hline
\end{tabular}

CI: confidence interval; F/M: female/male; ICU: intensive care unit; Min.: minimum; Max.: maximum; SD: standard deviation; ${ }^{*}$ during the postoperative 5day period according to the RIFLE (Risk-Injury-Failure-Loss-End stage) classification.

TABLe 2: Perioperative kidney function tests.

\begin{tabular}{|c|c|c|c|c|c|c|}
\hline Variable & $\begin{array}{c}\text { Pre-op, mean } \pm \\
\text { SD }\end{array}$ & $95 \% \mathrm{CI}$ & $\begin{array}{c}\text { PO 1st day, mean } \pm \\
\text { SD }\end{array}$ & $95 \% \mathrm{CI}$ & $\begin{array}{c}\text { PO 5th day, mean } \pm \\
\text { SD }\end{array}$ & $95 \% \mathrm{CI}$ \\
\hline BUN (mg/dL) & $40.4 \pm 13$ & $36.2-44.2$ & $41 \pm 15.9$ & $36-45.6$ & $40.1 \pm 18.4$ & $35.1-46.1$ \\
\hline Blood creatinine $(\mathrm{mg} / \mathrm{dL})$ & $1.1 \pm 0.2$ & $1-1.1$ & $1 \pm 0.3$ & $0.9-1$ & $1 \pm 0.3$ & $0.9-1.1$ \\
\hline Total urine output during $24 \mathrm{~h}(\mathrm{~mL})$ & $1909 \pm 1073$ & $1596-2258$ & $2885 \pm 822$ & $2630-3151$ & $2789 \pm 1444$ & $2360-3205$ \\
\hline $\operatorname{CCR}\left(\mathrm{mL} \cdot \mathrm{min}^{-1}\right.$ per $\left.1.73 \mathrm{~m}^{2}\right)$ & $85.7 \pm 30$ & $76.9-94.5$ & $109.4 \pm 42$ & $97.8-121.8$ & $87.8 \pm 32.8$ & $77.7-97.7$ \\
\hline $\operatorname{GFR}\left(\mathrm{mL} \cdot \mathrm{min}^{-1}\right.$ per $\left.1.73 \mathrm{~m}^{2}\right)$ & $77.5 \pm 18.9$ & $71.9-83.1$ & $89.7 \pm 27.9$ & $80.9-98.3$ & $81.2 \pm 19.7$ & $75.3-87.1$ \\
\hline
\end{tabular}

BUN: blood urea nitrogen; CCR: creatinine clearance rate; CI: confidence interval; GFR: estimated glomerular filtration rate measurement of the Cockcroft-Gault equation adjusted to the body surface area; Pre-op: preoperative; PO: postoperative; SD: standard deviation.

long-term $\mathrm{CPB}$ support, vasoconstriction, development of reperfusion injury, reduction of renal blood flow, presence of nonpulsatile flow, embolization of atherosclerotic plaque, perioperative hypothermia and perfusion-dependent cascade activation with extensive inflammatory response, and postoperative intra-aortic balloon pump usage $[5,16,18]$. Serum creatinine and estimated glomerular filtration rate calculations are generally used as comparison parameters in various retrospective studies in the current literature [5]. However, 24-hour creatinine clearance rate measurements as the basic comparative parameter could be more reliable. Besides, the reliability of estimated glomerular filtration rate calculations in predicting the actual creatinine clearance rate is not so high [10]. Therefore, we used 24-hour creatinine clearance rate measurements in the early postoperative period as the gold standard in addition to urinary output and creatinine criteria of the RIFLE as comparison parameters. Measurements such as serum creatinine, estimated glomerular filtration rate, or creatinine clearance rate (which requires 24-hour urine accumulation) reflect the onset of AKI late and require the development of biomarkers that detect acute kidney damage earlier [5]. Additionally, this requirement is more available for patients undergoing cardiac surgery under CPB where AKI is frequently encountered [19]. NGAL was identified as the biomarker with the highest positive predictive value alone in a study performed with many new biomarkers [20]. NGAL is a protein and a member of lipocalin family with a molecular weight of $25 \mathrm{kDa}[1,5]$. It is secreted from neutrophils and organ epithelium such as the kidney, uterus, prostate, and trachea [21]. Epithelial damage increases the serum and urinary NGAL levels. Infection, sepsis, and active inflammatory processes can also elevate the levels of NGAL. However, NGAL is less sensitive to infection and therefore not used in clinical practice in terms of infection [22, 23]. NGAL is rarely detected in plasma and urine in situations of healthy kidneys [5]. However, in cases of acute tubular injury, NGAL is detected both in serum and in urine and increases markedly [24]. NGAL is elevated as detectable in both serum and urine at 2 hours postischemically, and the elevation level correlates with the duration of renal ischemia [25]. NGAL reaches peak levels in both plasma and urine at 2-6 postoperative hours. Previous studies have shown that the AUC-ROC of the postoperative 2nd hour urinary NGAL measurement is between 0.85 and 0.871 in detecting the development of AKI after cardiac surgery $[1,20]$. Besides, the AUC-ROC of the plasma NGAL level for detecting AKI is 0.64. However, in this study, it appears that blood plasma samples are taken immediately after the termination of the CPB [26]. It should be noted that the levels of NGAL for blood serum and urine measurements reach peak levels within 2-4 hours. 


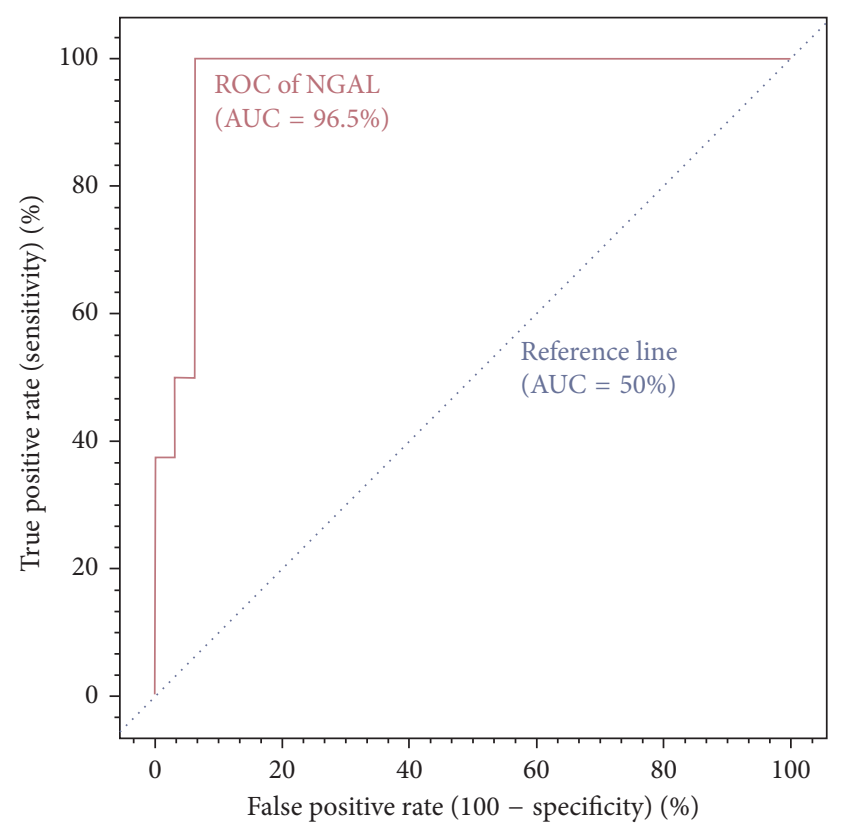

FIGURE 1: ROC curve of NGAL levels (cutoff value $=150 \mathrm{ng} / \mathrm{mL}$ ) on postoperative day 1 for diagnosis of kidney injury according to the RIFLE criteria. AUC: area under the curve; NGAL: neutrophil gelatinase-associated lipocalin; RIFLE: Risk-Injury-Failure-LossEnd stage; ROC: receiver-operating curve.

Therefore, the peak timing for NGAL measurements after cardiac surgery was usually at the time the patient was admitted to the intensive care unit $[1,9]$. It was predicted that NGAL may detected the CPB-related kidney dysfunction during the early intensive care unit period. As a result of a meta-analysis, it was stated that the AUC-ROC of plasma NGAL measurements for the detection of AKI was 0.71 for all studies and 0.73 for studies based on the RIFLE criteria [27]. In the previous studies performed, the sensitivity and specificity of plasma NGAL at postoperative 3 hours were $94.1 \%$ and $93.9 \%$, respectively [28]. By the help of the use of biomarkers that can detect AKI early, kidney damage can be reversed with effective interventions [8]. At this point, early detection of subclinical tubular injury is important [29]. NGAL can be used in the early detection of CPB-related AKI, thus making it possible to implement early interventional strategies. Current literature suggests that NGAL can be used as an early biomarker in the detection of acute kidney damage after CPB [5]. In parallel with these findings, we found that measurements of the plasma NGAL level on the first postoperative day could be used as an early biomarker in the detection of AKI after $\mathrm{CPB}$ with high sensitivity and specificity values in reflecting the period of postoperative five days.

\section{Conclusions}

It has been determined that plasma NGAL levels can be reliably used postoperatively for early diagnosis of kidney dysfunction in patients undergoing on-pump CABG. However, these promising results must be confirmed with further multicenter studies with larger patient populations.

\section{Additional Points}

Limitations of Our Study. The total number of patients enrolled in our study and retrospective, single-center conduction are major limitations of our study.

\section{Conflicts of Interest}

The authors have no conflicts of interest with this study.

\section{References}

[1] S. Liu, M. Che, S. Xue et al., "Urinary L-FABP and its combination with urinary NGAL in early diagnosis of acute kidney injury after cardiac surgery in adult patients," Biomarkers, vol. 18, no. 1, pp. 95-101, 2013.

[2] S. Uchino, J. A. Kellum, R. Bellomo et al., "Acute renal failure in critically ill patients: a multinational, multicenter study," JAMA, vol. 294, no. 7, pp. 813-818, 2005.

[3] C. Ronco, J. A. Kellum, and R. Bellomo, "Cardiac surgeryassociated acute kidney injury," The International Journal of Artificial Organs, vol. 31, no. 2, pp. 156-157, 2008.

[4] D. N. Wijeysundera, K. Karkouti, J. Y. Dupuis et al., "Derivation and validation of a simplified predictive index for renal replacement therapy after cardiac surgery," JAMA, vol. 297, no. 16, pp. 1801-1809, 2007.

[5] J. D. Kim, H. K. Chee, J. K. Shin et al., "Novel early predictor of acute kidney injury after open heart surgery under cardiopulmonary bypass using plasma neutrophil gelatinase-associated lipocalin," Korean Journal of Thoracic and Cardiovascular Surgery, vol. 47, no. 3, pp. 240-248, 2014.

[6] G. M. Chertow, E. Burdick, M. Honour, J. V. Bonventre, and D. W. Bates, "Acute kidney injury, mortality, length of stay, and costs in hospitalized patients," Journal of the American Society of Nephrology, vol. 16, no. 11, pp. 3365-3370, 2005.

[7] M. H. Rosner and M. D. Okusa, "Acute kidney injury associated with cardiac surgery," Clinical Journal of the American Society of Nephrology, vol. 1, pp. 19-32, 2006.

[8] M. Haase, R. Bellomo, P. Devarajan, P. Schlattmann, and A. Haase-Fielitz, "NGAL Meta-analysis Investigator Group. Accuracy of neutrophil gelatinase-associated lipocalin (NGAL) in diagnosis and prognosis in acute kidney injury: a systematic review and meta-analysis," American Journal of Kidney Diseases, vol. 54, no. 6, pp. 1012-1024, 2009.

[9] J. Mishra, C. Dent, R. Tarabishi et al., "Neutrophil gelatinaseassociated lipocalin (NGAL) as a biomarker for acute renal injury after cardiac surgery," The Lancet, vol. 365, no. 9466, pp. 1231-1238, 2005.

[10] O. O. Balkanay, D. Göksedef, S. N. Ömeroğlu, and G. İpek, "The reliability of estimated glomerular filtration rate in coronary artery bypass grafting," Turk Gogus Kalp Dama, vol. 24, no. 3, pp. 430-438, 2016.

[11] F. I. Fadel, A. M. Abdel Rahman, M. F. Mohamed et al., "Plasma neutrophil gelatinase-associated lipocalin as an early biomarker for prediction of acute kidney injury after cardiopulmonary bypass in pediatric cardiac surgery," Archives of Medical Science, vol. 8, no. 2, pp. 250-255, 2012.

[12] R. Bellomo, C. Ronco, J. A. Kellum, R. L. Mehta, and P. Palevsky, “Acute Dialysis Quality Initiative workgroup. Acute renal failure-definition, outcome measures, animal models, fluid therapy and information technology needs: the Second International Consensus Conference of the Acute Dialysis Quality Initiative (ADQI) Group," Critical Care, vol. 8, pp. R204-R212, 2004. 
[13] R. L. Mehta, J. A. Kellum, S. V. Shah et al., "Acute Kidney Injury Network: report of an initiative to improve outcomes in acute kidney injury," Critical Care, vol. 11, no. 2, p. R31, 2007.

[14] M. Haase, A. Haase-Fielitz, S. M. Bagshaw, C. Ronco, and R. Bellomo, "Cardiopulmonary bypass-associated acute kidney injury: a pigment nephropathy?," Contributions to $\mathrm{Ne}$ phrology, vol. 156, pp. 340-353, 2007.

[15] U. M. Fischer, W. K. Weissenberger, R. D. Warters, H. J. Geissler, S. J. Allen, and U. Mehlhorn, "Impact of cardiopulmonary bypass management on postcardiac surgery renal function," Perfusion, vol. 17, no. 6, pp. 401-406, 2002.

[16] G. M. Chertow, J. M. Lazarus, C. L. Christiansen et al., "Preoperative renal risk stratification," Circulation, vol. 95, no. 4, pp. 878-884, 1997.

[17] C. V. Thakar, S. Arrigain, S. Worley, J. P. Yared, and E. P. Paganini, "A clinical score to predict acute renal failure after cardiac surgery," Journal of the American Society of Nephrology, vol. 16, no. 1, pp. 162-168, 2005.

[18] K. R. Tuttle, N. K. Worrall, L. R. Dahlstrom, R. Nandagopal, A. T. Kausz, and C. L. Davis, "Predictors of ARF after cardiac surgical procedures," American Journal of Kidney Diseases, vol. 41, no. 1, pp. 76-83, 2003.

[19] D. P. Boer, Y. B. de Rijke, W. C. Hop, K. Cransberg, and E. M. Dorresteijn, "Reference values for serum creatinine in children younger than 1 year of age," Pediatric Nephrology, vol. 25, no. 10, pp. 2107-2113, 2010.

[20] M. Che, B. Xie, S. Xue et al., "Clinical usefulness of novel biomarkers for the detection of acute kidney injury following elective cardiac surgery," Nephron Clinical Practice, vol. 115, no. 1, pp. c66-c72, 2010.

[21] J. B. Cowland and N. Borregaard, "Molecular characterization and pattern of tissue expression of the gene for neutrophil gelatinase-associated lipocalin from humans," Genomics, vol. 45, no. 1, pp. 17-23, 1997.

[22] S. Y. Xu, K. Pauksen, and P. Venge, "Serum measurements of human neutrophil lipocalin (HNL) discriminate between acute bacterial and viral infections," Scandinavian Journal of Clinical and Laboratory Investigation, vol. 55, no. 2, pp. 125-131, 1995.

[23] L. Yan, N. Borregaard, L. Kjeldsen, and M. A. Moses, "The high molecular weight urinary matrix metalloproteinase (MMP) activity is a complex of gelatinase B/MMP-9 and neutrophil gelatinase-associated lipocalin (NGAL): modulation of MMP-9 activity by NGAL," Journal of Biological Chemistry, vol. 276, no. 40, pp. 37258-37265, 2001.

[24] D. R. Mcllroy, G. Wagener, and H. T. Lee, "Biomarkers of acute kidney injury: an evolving domain," Anesthesiology, vol. 112, no. 4, pp. 998-1004, 2010.

[25] J. Mishra, Q. Ma, A. Prada et al., "Identification of neutrophil gelatinase-associated lipocalin as a novel early urinary biomarker for ischemic renal injury," Journal of the American Society of Nephrology, vol. 14, no. 10, pp. 2534-2543, 2003.

[26] T. E. Perry, J. D. Muehlschlegel, K. Y. Liu et al., "Plasma neutrophil gelatinase-associated lipocalin and acute postoperative kidney injury in adult cardiac surgical patients," Anesthesia \& Analgesia, vol. 110, no. 6, pp. 1541-1547, 2010.

[27] J. Ho, N. Tangri, P. Komenda et al., "Utility for predicting acute kidney injury associated with cardiac surgery in adults: a meta-analysis," American Journal of Kidney Diseases, vol. 66, no. 6, pp. 993-1005, 2015.

[28] T.A. Ghonemy and G. M. Amro, "Plasma neutrophil gelatinaseassociated lipocalin (NGAL) and plasma cystatin C (CysC) as biomarker of acute kidney injury after cardiac surgery," Saudi
Journal of Kidney Diseases and Transplantation, vol. 25, no. 3, pp. 582-588, 2014.

[29] M. Haase, R. Bellomo, C. Albert et al., "The identification of three novel biomarkers of major adverse kidney events," Biomarkers in Medicine, vol. 8, no. 10, pp. 1207-1217, 2014. 


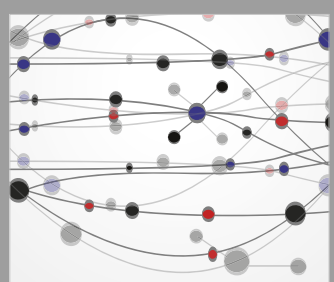

The Scientific World Journal
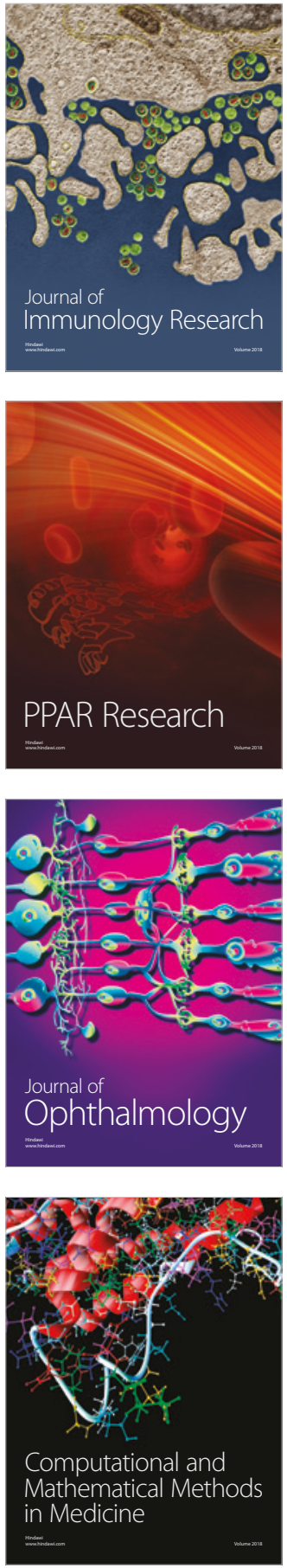

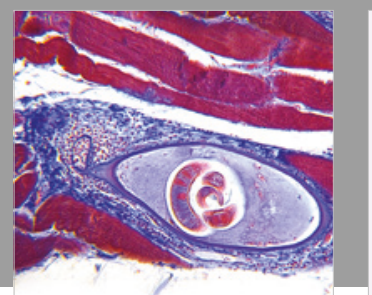

Gastroenterology Research and Practice

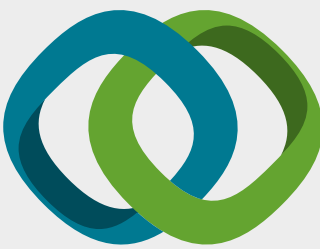

\section{Hindawi}

Submit your manuscripts at

www.hindawi.com
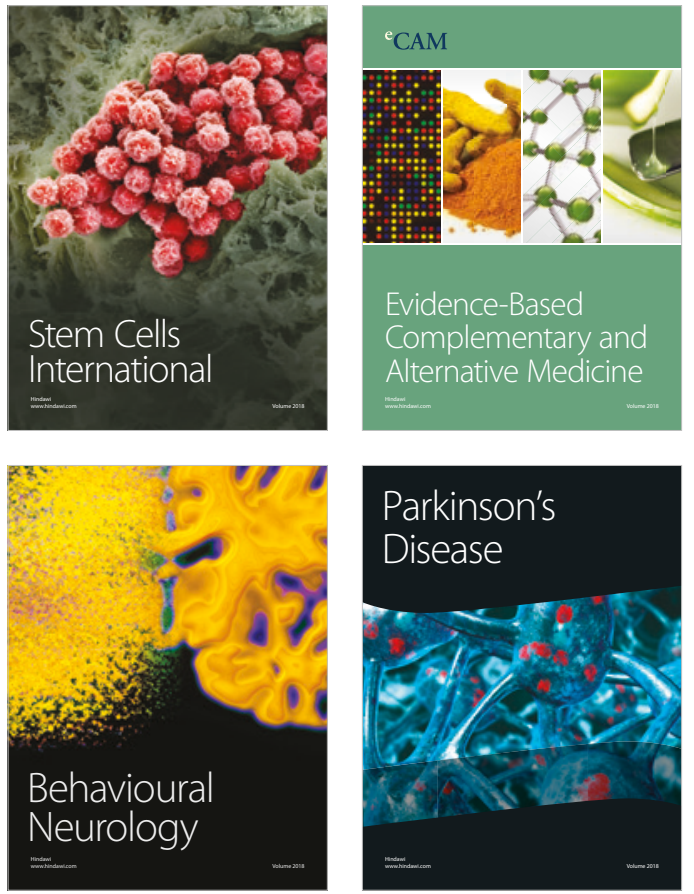

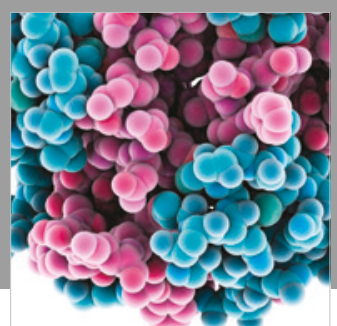

ournal of

Diabetes Research

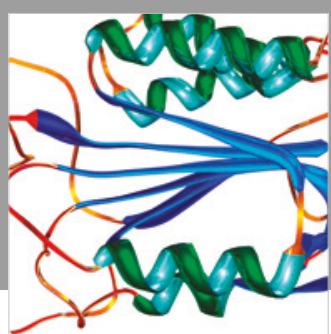

Disease Markers
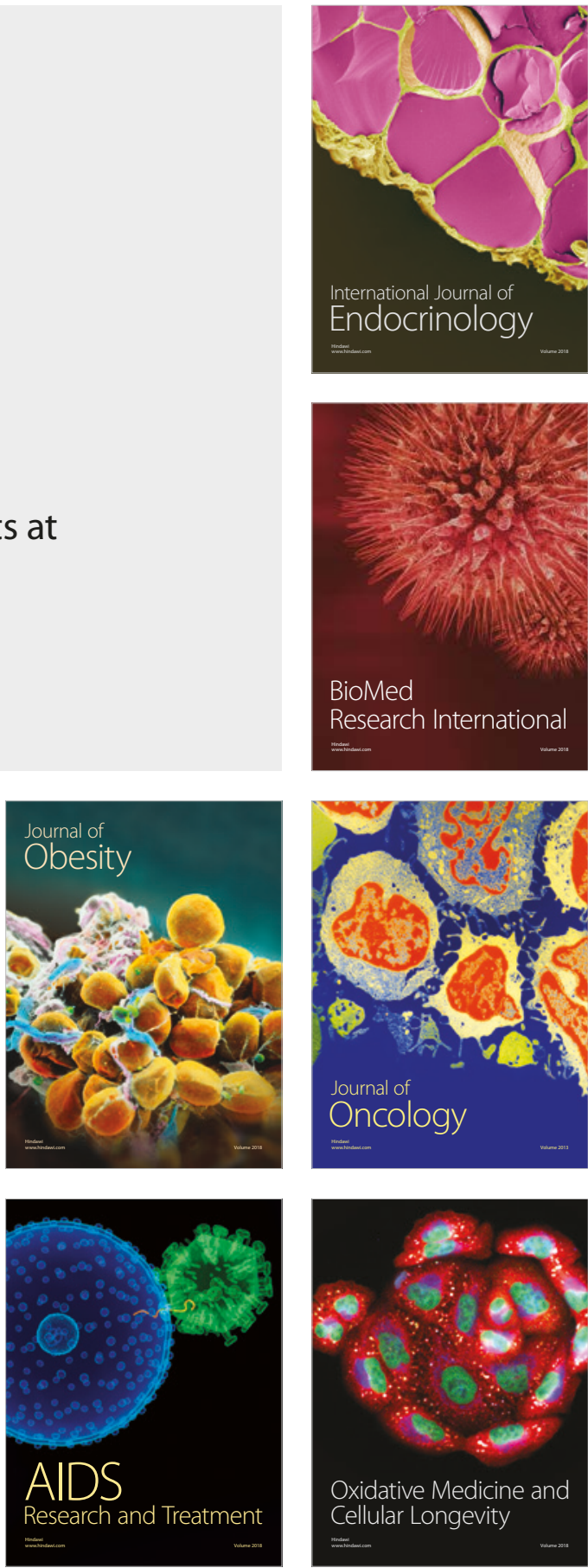